\title{
Preparation and Performance Research of WPU Modified by Nanopoly- phosphazenes
}

\author{
Chen Jianbing*, Ning Xiangli, Hu Wenjing and Wang Jiangjie \\ Department of Materials and Chemistry Engineering, Chizhou University, Chizhou, 247000, P.R. China
}

\begin{abstract}
It is reported in this paper that waterborne polyurethane (WPU) can be modified by nanopolyphosphazenes fibers, which had been prepared containing hydroxyl groups, followed by reaction with WPU. At the same time, physical blends had also been carried out in the experiments. The results showed that the tensile strength of the WPU films through chemical modification containing $0.5 \mathrm{wt} \%$ fibers is, $1.22 \mathrm{MPa}$, and that the elongation at break is $1294.50 \%$. This data has significantly increased when compared against WPU, in which the elongation at break is only $785.50 \%$. The thermal decomposition temperature of the WPU modified by nanopolyphosphazenes is $50 \%$ higher than that of regular WPU. At the same time, its preparation method had some influences on the mechanical properties of the composite materials.
\end{abstract}

Keywords: Modification, nanopolyphosphazenes, preparation, waterborne polyurethane.

\section{INTRODUCTION}

Waterborne resins forming a non-sticking surface have been investigated because of their maintenance-free material demands, as well as emphasis on the reduction of solvent emissions. Waterborne polyurethane, which the organic solvent is replaced by water as a dispersion medium, has been used in textile, leather, paper and the other fields. WPU has excellent dispersion stability and good film-forming properties. However, there are still some inevitable defects of WPU, its modification also become a hot research topic [1-3]. The improvement methods of WPU have been widely reported [4-9]. Polyphosphazenes are phosphorus and nitrogen's compounds with alternating single and double bonds [10]. Therefore, the main chain of polyphosphazenes is very soft and is mainly used in biological medicine, aerospace materials, flame retardants and so on [11]. So, WPU modified by nanopolyphosphazenes fibers are investigated in this paper.

\section{EXPERIMENTAL}

\subsection{Materials}

Hexachloro cyclotriphosphazene (HCCP, 99\%), 4,4'nanomaterials dihydroxy diphenyl sulfone (BPS, 99\%) and 2, 2 - (hydroxymethyl) propionic acid (DMPA, 98\%) were supplied by Shanghai Jichun reagent Co., Ltd.; acetone (A.R) was supplied by Shanghai Runjie chemical reagent Co., Ltd.; tetrahydrofuran (A.R) was supplied by Tianjin Bodi chemical reagent Co., Ltd.; polyether glycol (PPG2000, $\mathrm{Mn}=2000$ ) was supplied by Sangsu Haian Petroleum chemical Plant; and isophorone diisocyanate (IPDI, 99\%) was supplied by Shanghai Hansi chemical reagent Co., Ltd.

*Address correspondence to this author at the Department of Materials and Chemistry Engineering, Chizhou University, Chizhou, P.R. China; Tel: +865662748625; E-mail: chjb8008@163.com

\subsection{General Procedure}
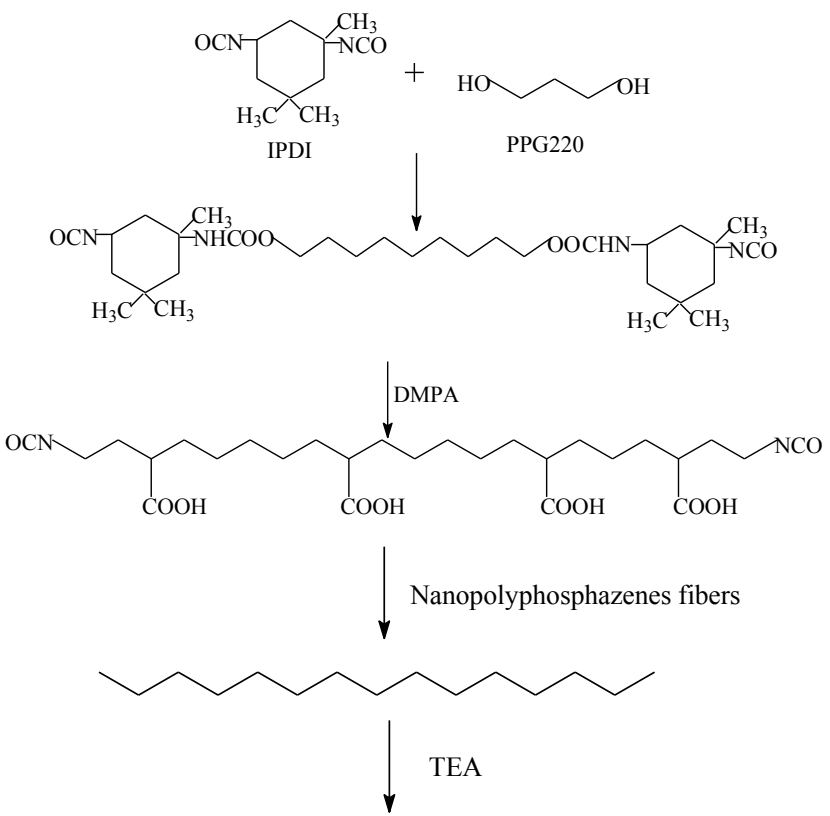

Composite materials of WPU modified nanopolyphosphazenes

Synthetic route of WPU modified by nanopolyphosphazenes.

\subsection{The Synthesis of Nanomaterials Polyphosphazenes}

According to the raw material mole ratio (HCCP: BPS: TEA $=1: 3: 9)$, the concentration of the HCCP is about 4.5 to $5.5 \mathrm{~g} / \mathrm{L}$, of which acetone was selected as solvent, and then polyphosphazenes had been prepared by ultrasonic cleaning machine, and the reaction temperature is about $10^{\circ} \mathrm{C}$, and the power transferred is about 40-50 KHZ. At the end, some nanofiber samples of polyphosphazenes can be obtained, which is the white powder solid. 


\subsection{WPU Modified by Nanopolyphosphazenes}

As shown in Section 2.2, according to Mole ratio of IPDI:PPG2000: DMPA=2.5: 1: 1 , the composite materials of WPU modified nanopolyphosphazenes had been prepared by chemical reaction, which the nanopolyphosphazenes had been added with $0.5 \%$ and $1 \%$ of the content of the WPU mass. At the same time, the pure WPU had also been prepared, and blended physically with the nanopolyphosphazenes.

\subsection{Detection Method}

WPU had been put into the glassware, and then it would be put into the constant temperature vacuum drying oven for $12 \mathrm{~h}$ after room temperature drying. After cooling the WPU films would be removed into the oven at room temperature. The films of WPU modified nanopolyphosphazenes by chemical reaction and blended physically had also been prepared. Nanopolyphosphazenes had been analyzed by the fourier transform infrared spectrometer (NEXUS-870, Thermo Nicolet, USA) at room temperature, with scanning wave number range $500-4000 \mathrm{~cm}^{-1}$, and scanning interval 44 $\mathrm{cm}^{-1}$. The morphology of the nanopolyphosphazenes had been observed by scanning electron microscope (S4800 type, HITACHI, Japan), and the samples had been sprayed by gold. The thermostability of films had been tested by the thermogravimetric analyzer (HTG - type, Beijing Henjiu scientific instrument, China) at room temperature. The temperature range is $0-900^{\circ} \mathrm{C}$, and the heating rate is $20^{\circ} \mathrm{C} / \mathrm{min}$. Mechanical properties were investigated by a universal testing machine that is DELL-20000 tensile tester supplied by Shanghai Dengjie Machine Co., Ltd according to GB/T528 - 2009. Experiments were conducted in laboratory air at room temperature and relative humidity of about $50 \pm 10 \%$, the tensile speed of $5 \mathrm{~mm} / \mathrm{min}$; dumbbell specimens were prepared by omnipotent sample maker.

\section{RESULTS AND DISCUSSION}

\subsection{FTIR Analysis}

As shown in Fig. (1), the vibration absorption peak of $\mathrm{OH}$ and $-\mathrm{P}=\mathrm{N}-$ can be observed in (a), which are $-3427 \mathrm{~cm}^{-1}$ and $1381 \mathrm{~cm}^{-1}$ respectively, the vibration absorption peak of -NHCOO-, -C-O-C- and -C-N can be observed in (b), which are $1631 \mathrm{~cm}^{-1}, 1383 \mathrm{~cm}^{-1}$ and $1119 \mathrm{~cm}^{-1}$ respectively. FTIR of WPU modified by nanopolyphosphazenes fibers can be seen in Fig. (1c), the vibration absorption peak of -NCO cannot be found. It could be inferred from the fact that there is a chemical reaction between waterborne polyurethane and nanopolyphosphazenes through $-\mathrm{NCO}$ and $-\mathrm{OH}$.

\subsection{Morphology Analysis of Nanopolyphosphazenes}

As shown in Fig. (2), it can be seen that there is a large number of irregular silk fibers with tangles, in which the diameter of those fibers is about $50-200 \mathrm{~nm}$. It could therefore, be inferred that nanopolyphosphazenes in the synthetic process had some influence by ultrasonic separation, which resulted in the small size of nanopolyphosphazenes that helped progress the polymerization at a continued pace. (a) Nanopolyphosphazenes fibers

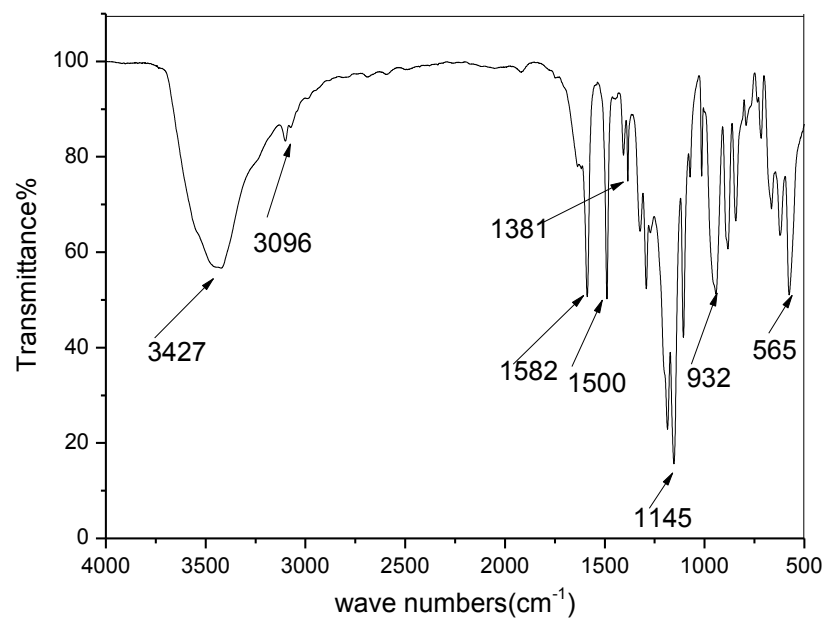

(b) WPU

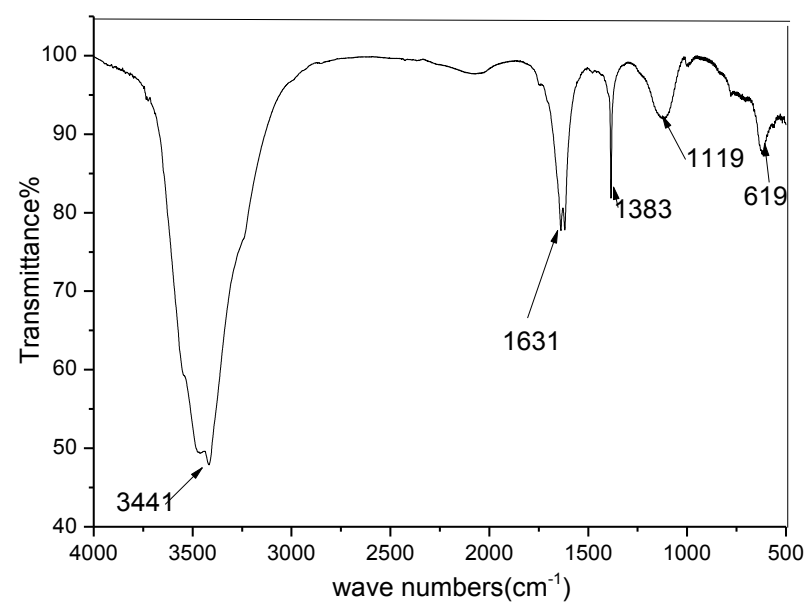

(c) WPU modified by nanopolyphosphazenes fibers (1.0wt \%)

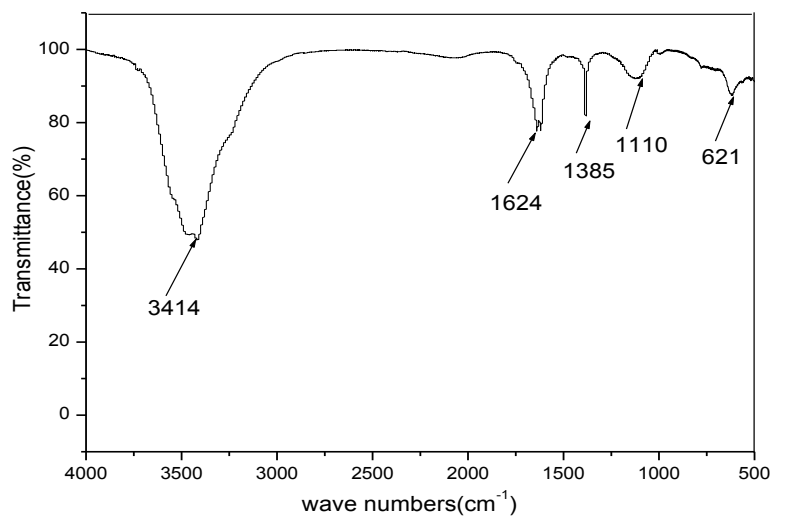

Fig. (1). FTIR of nanopolyphosphazenes and WPU modified by nanopolyphosphazenes fibers.

\subsection{Thermostability}

As shown in Fig. (3), the films of WPU and WPU modified by nanopolyphosphazenes fibers had been analyzed by thermogravimetry. It can be observed that: (a) when the 


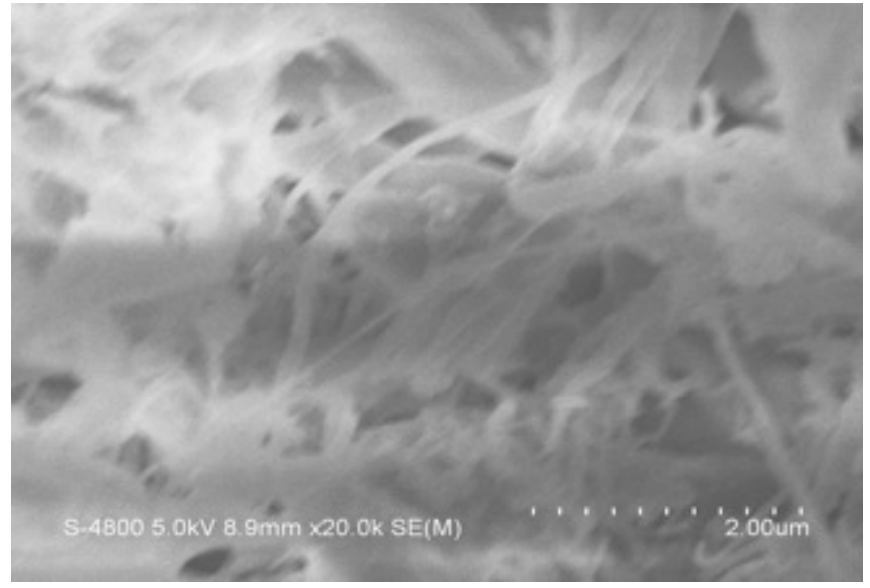

Fig. (2). SEM of nanopolyphosphazenes fibers.

(a) WPU

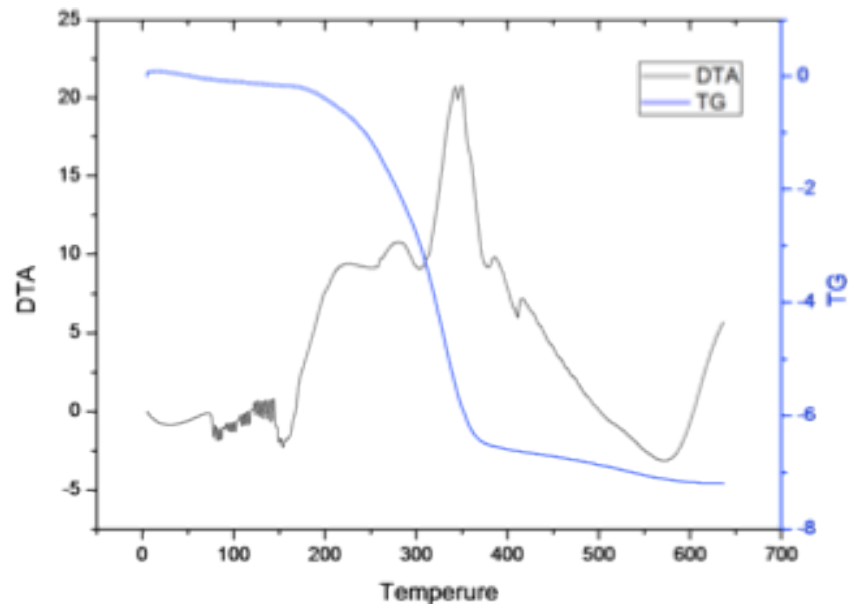

(b) WPU modified by nanopolyphosphazenes fibers

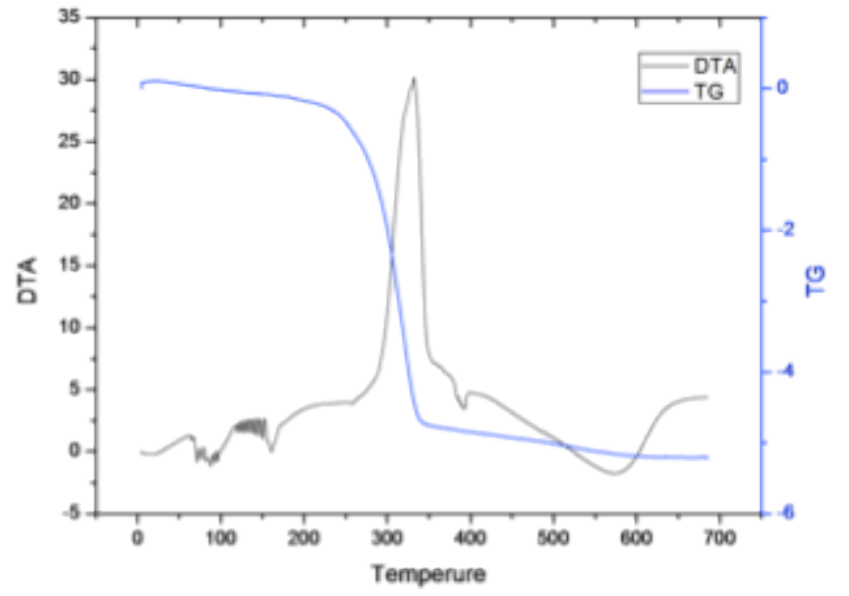

Fig. (3). TGA curves of WPU and WPU modified by nanopolyphosphazenes fibers (1.0wt \%).

thermal decomposition temperature of WPU is about $200^{\circ} \mathrm{C}$. With the rise of temperature, the thermal pyrolysis is obvious compared to before the decomposition temperature, of which the first thermal pyrolysis is about $155^{\circ} \mathrm{C}$; (b) when the thermal decomposition temperature of WPU modified by nanopolyphosphazenes fibers is about $250^{\circ} \mathrm{C}$. With the rise of temperature, the thermal pyrolysis almost did not exist compared to before the decomposition temperature. Therefore, it can be inferred that thermostability of the composite materials of WPU modified by nanopolyphosphazenes could be much improved compared to that of WPU.

\subsection{Mechanical Properties}

Table 1 shows the data tensile strength versus elongation at break of WPU and WPU modified by different contents of nanopolyphosphazene fibers. The tensile strengths respectively $0.5 \mathrm{wt} \%$ and $1 \mathrm{wt} \%$ of nanopolyphosphazenes fibers/WPU are all 1.22 MPa, in which the tensile strength of modified WPU is less than that of WPU. But the elongation at break had been improved obviously by modification, which has achieved a value up to $1294.50 \%$. With the increase of nanopolyphosphazene fibers, the elongation at break of WPU modified by nanopolyphosphazenes fibers decreased, which shows that the toughness of WPU can be improved by adding an appropriate amount of nanopolyphosphazene fibers. So the waterborne polyurethane material modified by nanopolyphosphazenes is more suitable for applications in manufacturing high elastic film products.

In order to study the composite methods, nanopolyphosphazenes fibers and WPU had been blended physically, and the tensile strengths of $1.0 \mathrm{wt} \%$ and $1.5 \mathrm{wt} \%$ nanopolyphosphazene fibers/WPU are about $1 / 6$ of that of WPU, which is also lower than that after the chemical modification. However, the elongation at break obviously increased. Therefore, it is concluded that the composite methods of WPU and nanopolyphosphazenes would influence the mechanical properties.

\section{CONCLUSION}

The nanopolyphosphazene fibers and the composite materials of WPU modified by fibers had been prepared in the experiments; the size of nanopolyphosphazene fibers is about $50-200 \mathrm{~nm}$.

The thermal decomposition temperature of WPU modified by nanopolyphosphazenes fibers is about $250^{\circ} \mathrm{C}$, which is higher than that of WPU.

The toughness of WPU can be improved through modification. The elongation at break of WPU modified by nanopolyphosphazenes fibers is up to $1294.50 \%$. At the same time, the composite methods of WPU and nanopolyphosphazenes would influence the mechanical properties.

\section{CONFLICT OF INTEREST}

The authors confirm that this article content has no conflict of interest.

\section{ACKNOWLEDGEMENTS}

This work was financially supported by the Department of Education (KJ2013A197), the Department of Science and Technology (1403062016) in Anhui Province and College 
Table 1. Mechanical parameters of WPU and WPU modified by nanopolyphosphazenes.

\begin{tabular}{|c|c|c|}
\hline Samples & Tensile Strength (MPa) & Elongation at Break (\%) \\
\hline WPU & 2.40 & 785.50 \\
\hline $0.5 \mathrm{wt} \%$ nanopolyphosphazenes fibers/WPU (reaction) & 1.22 & 1294.50 \\
\hline $1.0 \mathrm{wt} \%$ nanopolyphosphazenes fibers/WPU (reaction) & 1.22 & 1194.42 \\
\hline $1.0 \mathrm{wt} \%$ nanopolyphosphazenes fibers/WPU (blend) & 0.41 & 1534.60 \\
\hline $1.5 \mathrm{wt} \%$ nanopolyphosphazenes fibers/WPU (blend) & 0.40 & 1540.70 \\
\hline
\end{tabular}

students' scientific and technological innovation projects (201211306012, 201311306039).

\section{REFERENCES}

[1] Chen YB, Zhang XY, Dai JB. Synthesis and characterization of PDMS modified UV-curable waterborne polyurethane dispersions for soft tact layers. Prog Org Coat 2007; 60: 63-8,

[2] Hua FT, Yi X, Wang L, Zhang CQ, Xian ZZ. Improved flexibility and water resistance of soy protein thermoplastics containing waterborne polyurethane. Indust Crops Prod 2010; 32: 13-20.

[3] Yu HG, Shu C, Li GS, Wang W, Ma ZH. Waterborne polyurethane/poly (n-butyl acrylate-styrene) hybrid emulsions: Particle formation, film properties, and application. Prog Org Coat 2012; 74: 248-56.

[4] Lung $\mathrm{H}$, Wang $\mathrm{CM}, \mathrm{Fu} \mathrm{AG}$, Wen TC. Frequency dependent conductivity of the thin film blend of electroluminescent poly (pphenylene vinylene) with waterborne polyurethane as ionomer. Thin Solid Films 2004; 466: 197-203.

[5] Dao X, Sun X, Miao KJ, Zhang H, Kim Y, Yuan A. Forming waterborne polyurethane composites fabricated with silane coupling agent functionalized nano-silica. J Colloid Interface Sci 2011; 276: 68-80.

[6] Zhang B, Yu NT, Long YY, Moussy FM. A long-term flexible minimally-invasive implantable glucose biosensor based on an epoxy-enhanced polyurethane membrane. Biosens Bioelectron 2006; 21: 2275-82

[7] Xiao V, Gao YC, Zhu SZ, Wei GZ, Zhou WB. Preparation and characterization of well-dispersed waterborne polyurethane/ CaCO3, nanocomposites. Colloid Surf A 2011; 377: 312-7.

[8] Chen JB, Guo Q, Sun JL. A study on linseed oil modified waterborne polyurethane coatings. Mat Sci Forum 2011; 686: 52832.

[9] Zhang SG, Guo Q, Zhao ZP. Microcrystalline cellulose modified waterborne polyurethane. Mater Perform 2012; 51: 35-9.

[10] $\mathrm{Fu} \mathrm{JW}, \mathrm{Chen} \mathrm{ZM}, \mathrm{Xu}$ Q. The production of porous carbon nanofibers from cross-linked polyphosphazene nanofibers. Carbon 2011; 49: 1037-9.

[11] Fu JW, Huang YW, Pan Y. An attempt to prepare carbon nanotubes by carbonizing polyphosphazene nanotubes with high carbon content. Mater Lett 2008; 62: 4130-3.

This is an open access article licensed under the terms of the Creative Commons Attribution Non-Commercial License (http://creativecommons.org/licenses/ by-nc/4.0/) which permits unrestricted, non-commercial use, distribution and reproduction in any medium, provided the work is properly cited. 